\section{The Neutron as a Quantum Object}

\author{
Helmut Rauch \\ Atominstitut der Österreichischen Universitäten, A-1020 Wien
}

Neutron interference experiments elucidate various properties of matter waves and permit a novel kind of wave function tomography.

Quantum and relativity theory are still the basis for our understanding of nature. Quantum optics is part of it and contributed substantially to that understanding, due to progress in laser and quantum state preparation techniques. An extension to quantum particle optics electrons, neutrons, atoms and molecules makes this field of research even more attractive. In every case interference experiments yield the deepest insight into the miracles of nature.

Two decades ago the first perfect crystal neutron interferometer was tested by an Austrian-German cooperative group at the $250 \mathrm{~kW}$ TRIGA-reactor in Vienna [1]. Since then neutron interferometry has become a laboratory for quantum mechanical testing. Widely separated coherent beams of thermal neutrons $(\lambda \approx 1.8 \AA$, $E \approx 0.025 \mathrm{eV}$ ) were produced by dynamic Laue-reflection in an appropriately shaped perfect silicon crystal (Fig. 1). Analogies to the Mach-Zehnder type interferometers used in light optics, and to the Bonse-Hart interferometers [2] developed for X-rays, are obvious. Electron and the recently developed atom interferometery use somewhat similar schemes [3].

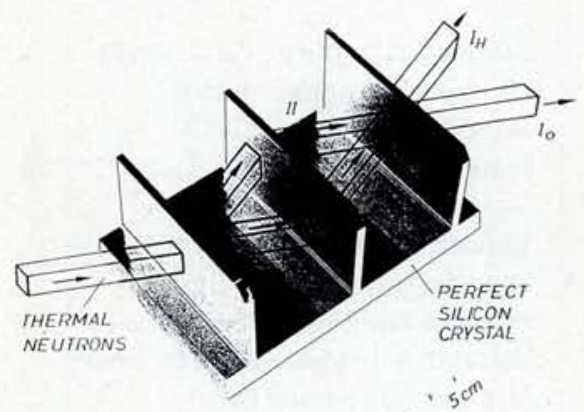

Fig. 1: Perfect crystal neutron interferometer. The monolithic design provides the parallelism of the reflecting lattice planes to within one lattice constant. The whole system represents a macroscopic quantum device with a wide beam separation.
Neutrons are massive and extended quantum objects. They are Fermions with an internal structure comprising one up- and two down-quarks. Inside a magnetic field, the magnetic moment of the neutron gives rise to a two energy level system between which transitions can be induced by specific oscillating magnetic fields. Neutrons are subject to strong, electromagnetic and gravitational interactions as well as topological phenomena, which all cause measurable interference effects. Following the complementarity principle of quantum mechanics, the neutron behaves purely as a wave inside the interferometer.

It follows, from general symmetry considerations, that the wave functions originating from beam paths I and II and composing the forward beam (o) behind the interferometer are equal in amplitude and phase because they are transmittedreflected-reflected (TRR) and reflectedreflected-transmitted (RRT), respectively. Different kinds of interaction can cause phase shifts between the coherent beams which can be calculated by the path integral of the canonical momentum $\mathbf{k}_{\mathrm{c}}$ along the interferometer loop, $\chi=\oint \mathbf{k}_{\mathrm{c}} \mathrm{d} \mathbf{s}=\boldsymbol{\Delta} \cdot \mathbf{k}$.

The ideal interference pattern is therefore given by:

$$
\begin{aligned}
\mathrm{I}(\boldsymbol{\Delta}) & \propto\left|\psi^{\mathrm{I}}(0)+\psi^{\mathrm{II}}(\boldsymbol{\Delta})\right|^{2} \\
& \propto 1+|\Gamma(\boldsymbol{\Delta})| \cos \boldsymbol{\Delta} \cdot \mathbf{k}
\end{aligned}
$$

where $\boldsymbol{\Delta}$ denotes the spatial shift of the interfering wave packets which is determined by the refracive index of the phase shifting material or field, and which also defines the phase shift $\chi$ of the interfering beams $(\chi=\boldsymbol{\Delta} \cdot \mathbf{k})$. In the case of a pure nuclear phase shifter, one gets $\chi=-N b_{c} \lambda D$, where $\mathrm{N}$ denotes the particle density, $\mathrm{D}$ the thickness, $b_{c}$ the coherent scattering length, and $\lambda$ the wavelength of the neutrons. $\Gamma(\boldsymbol{\Delta})$ denotes the coherence function which (as in light optics) is related to the momentum distribution function of the beam $\mathrm{g}(\mathbf{k})$

$$
\Gamma(\Delta) \propto \int g(\mathbf{k}) \mathrm{e}^{\mathrm{i} \mathbf{k} \boldsymbol{d}} \mathrm{dk} .
$$

$|\Gamma(\boldsymbol{\Delta})|$ approaches 1 for $\boldsymbol{\Delta} \rightarrow 0$ and zero for $\boldsymbol{\Delta} \rightarrow \propto$. Its characteristic spatial dimension determines the coherence lengths $\Delta_{i}{ }^{c}$, which, for Gaussian shaped beams, are directly related to the momentum distribution of the beam $\delta \mathrm{k}_{\mathrm{i}}$ by the Heisenberg uncertainty relation $\Delta_{i}^{\mathrm{c}} \delta \mathrm{k}_{\mathrm{i}}=$ $1 / 2$.

All neutron interference experiments pertain to the domain of self-interference, where, in nearly all cases, only one neutron is inside the interferometer while the next one has yet to be born and is still contained in the uranium nuclei of the reactor fuel. Although there is no interaction between different neutrons, they have a certain common history within predetermined limits which are defined, e.g., by the neutron moderation process, their movement along neutron guide tubes, the monochromator crystal and the special interferometer setup. Therefore, any real interference pattern contains single particle as well as ensemble properties. Minor deviations of the interference pattern from Eq. (1) may occur due to slight imperfections of the experimental setup. With a well balanced interferometer, a contrast of up to $90 \%$ can be achieved and more than the 300 th order of interference can be observed. This provides the basis for various coherence experiments and for precise measurements of scattering lengths. Here we focus on more fundamental experiments, proving various intrinsic properties of quantum mechanics.

The most important experiment was probably the verification of the $4 \pi$ symmetry of spinor wave functions which was achieved independently in Europe and the U. S. soon after the successful operation of perfect crystal interferometers [4]. For the propagation of the wave function inside a magnetic field, which couples to the magnetic moment of the neutrons ( $\mathrm{H}$ $=-\boldsymbol{\mu B})$, one gets

$$
\psi \rightarrow \psi_{0}{ }^{-\mathrm{i}(\mathrm{H} / / h)}=\psi_{0} \mathrm{e}^{-\mathrm{i} \sigma \alpha / 2}=\psi(\alpha),
$$

where $\boldsymbol{\sigma}$ are the Pauli-spin matrices and $\alpha$ is the Larmor rotation angle around the field $(\alpha=(2 \mu / h v) \oint$ Bds; $\mu=$ magnetic moment, $\mathrm{v}=$ neutron velocity, $\mathrm{B}=$ magnetic field strength). Thus, one gets

$$
\begin{aligned}
& \psi(2 \pi)=-\psi(0) \\
& \psi(4 \pi)=\psi(0)
\end{aligned}
$$

which becomes measurable by the interference pattern at low order of interference 
$\mathrm{I} \propto|\psi(0)+\psi(\alpha)|^{2} \propto 1+\cos (\alpha / 2)$

(5)

in excellent agreement with the experimental observation (Fig. 2). The $4 \pi-$ periodicity effect has been observed both for unpolarized and polarized neutrons, which demonstrates the intrinsic feature of this $4 \pi$-symmetry phenomenon and that single particle and not only ensemble properties are described by the wave function.

Extending this kind of investigation, the quantum mechanical spin-superposition law has been verified on a macroscopic scale with polarized incident neutrons split coherently and polarizationinverted in one beam path (i.e. Larmor angle rotated by $\alpha=\pi$ ), giving a wave function for the forward beam:

$$
\psi \propto \psi^{\mathrm{I}}+\psi^{\mathrm{II}} \propto\left(|z\rangle+\mathrm{e}^{\mathrm{i} x}|-\mathrm{z}\rangle\right)
$$

and a final polarization in the $\mathrm{x}, \mathrm{y}$-plane, $\mathrm{P}=(\cos \chi, \sin \chi, 0)$ i.e. perpendicular to both states before superposition [5]. Eugene Wigner pointed out that, in this case, a pure initial state in the $|z\rangle$-direction is transformed into a pure state in the

Fig. 2: $4 \pi$-symmetry of spinor wave functions. For a $2 \pi$ rotation, a sign change of the wave function occurs, which is a measurable and not a hidden quantity in quantum mechanics. The interferograms are shifted compared to Eq. (5) due to an intrinsic phase of the setup.
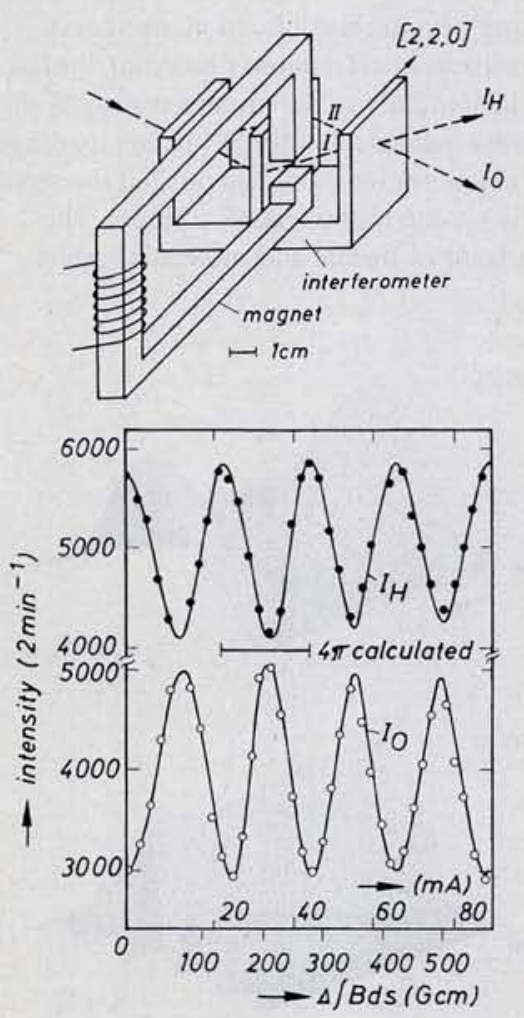

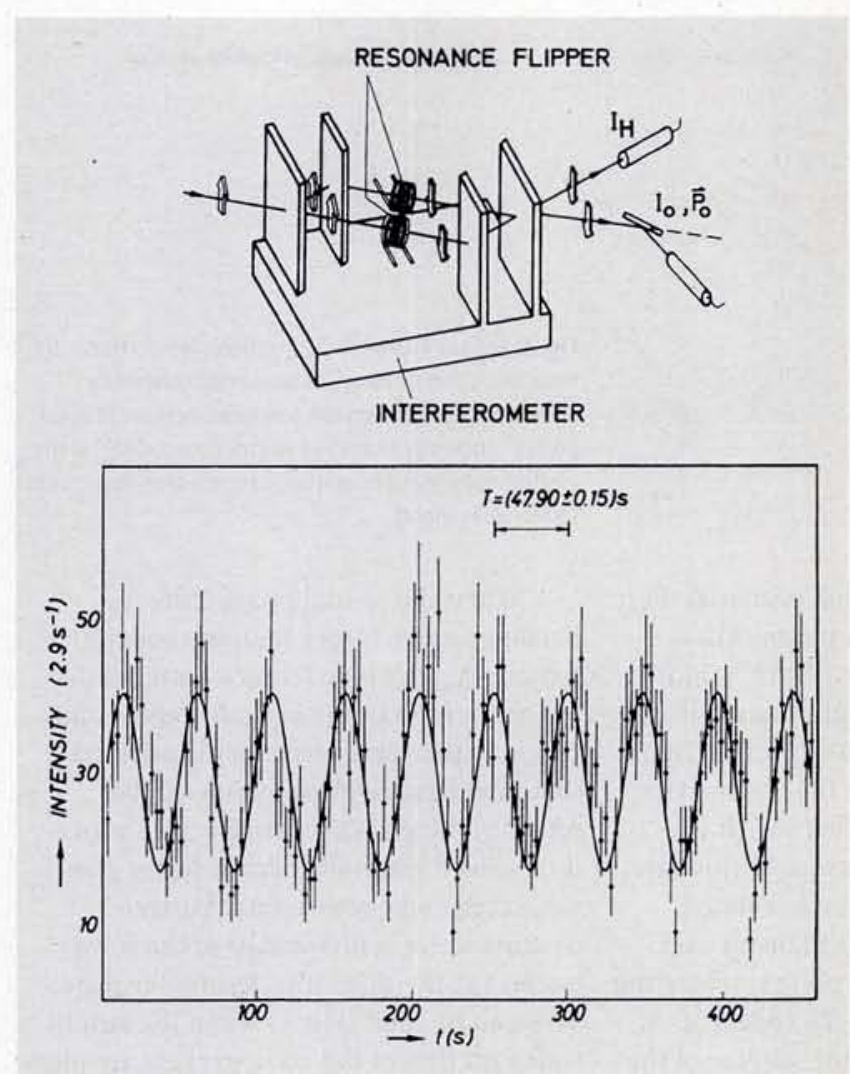

Fig. 3: Magnetic Josephsoneffect. Slightly different energy transfers within resonance flipper cavities in both coherent beams cause a macroscopic time modulation of the outgoing beams which exhibit an extremely high energy sensitivity up to $2.7 \times 10^{-19} \mathrm{eV}$. $|x\rangle$-direction $(\chi=0)$, although in one beam path no spin reversal $(|z\rangle)$ and in the other a complete spin reversal (-|z ) occurs.

When the spin reversal is accomplished by a Rabi-type resonance flipper, the total energy changes by the amount of the Zeeman energy $2 \mu \mathrm{B}_{0}=\hbar \omega_{\mathrm{L}}$, causing a time-dependent phase shift $\left(\omega_{L} \cdot t\right)$ and a final polarization, rotating with the Larmor frequency $\omega_{\mathrm{L}}$ in the $\mathrm{x}, \mathrm{y}$-plane without being driven by a magnetic field. A slight modification of this kind of experiment permitted the verification of the magnetic Josephson effect [6] (Fig. 3). In this case, Rabi-flippers are operated in both beam paths but with slightly different Larmor resonance frequencies, $\omega_{\mathrm{L}}{ }^{(1)}$ and $\omega_{L}{ }^{(2)}$, due to slightly different guide fields, $\mathrm{B}_{0}{ }^{(1)}$ and $\mathrm{B}_{0}{ }^{(2)}$, respectively. This causes a temporal beam modulation in the form of a quantum beat effect

$$
I \propto 1+\cos \left[\chi+\left(\omega_{\mathrm{L}}^{(1)}-\omega_{\mathrm{L}}{ }^{(2)}\right) \mathrm{t}\right]
$$

where the time-dependent phase shift is driven by the magnetic interaction

$$
\Delta(\mathrm{t})=\left(\omega_{\mathrm{L}}{ }^{(1)}-\omega_{\mathrm{L}}{ }^{(2)}\right) \mathrm{t}=2 \mu \Delta \mathrm{B}_{0} \mathrm{t} / \hbar .
$$

The observed period of the beam modulation and the energy sensitivity are related through the quantum mechanical uncer- tainty relation. The modulation is driven by an energy difference of $\Delta \mathrm{E}=8.6 * 10^{-17}$ $\mathrm{eV}$ and the energy sensitivity is even $2.7 * 10^{-19} \mathrm{eV}$. This phenomenon is analogous to the well-known electric Josephson effect, where the phase shift in a superconducting tunnel junction is driven by the electric interaction $\Delta_{l}(\mathrm{t})=2 \mathrm{eVt} / \hbar$.

All the phenomena described above are closely connected to the topological effects such as the geometric Berry phase [7] when the magnetic field is twisted or when the rotation is done around various axes, or the scalar Aharonov-Bohm effect when the magnetic field is switched on and off while the neutron is inside the field region [8]. This shows various additional features of the wave function which, for a long time, did not appear to be accessible by experiment. In all these cases, the canonical momentum - not the kinetic one $(\mathrm{k} \hbar=\mathrm{mv})$ - changes, and therefore no classical force acts on the particle. Figure 4 depicts this situation for charged particles (electrons) and for neutrons.

The coupling of the neutron to the gravitational field has also attracted much interest because the unification of quantum and gravitational theory is still outstanding. The action of the earth's gravitational field appears when the interferometer crystal is rotated (angle $\varepsilon$ ) around a horizontal axis and one beam path experi- 


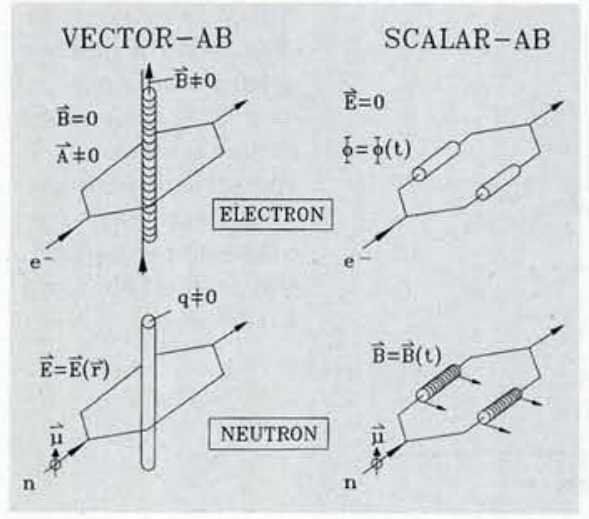

ences a higher gravitational potential than the other [9]. The related phase shift is given by $\chi_{g}=m^{2} g \lambda A \sin \varepsilon /\left(2 \pi \hbar^{2}\right)$, indicating coupling between gravitational and quantum mechanical units. Neutron interferometry also permitted the observation of the neutron Sagnac-effect which is caused by the earth's rotation. In this case, the interferometer crystal was rotated (angle $\varepsilon$ ) around the vertical beam axis (i.e. towards the cardinal points) where the enclosed area $\mathrm{A}$ between the coherent beams acts like a flag on the surface of the rotating earth (frequency $\omega$ ). The related phase shift can be written as $\chi_{\mathrm{s}}=2 \mathrm{m \omega A}$ $\sin \phi_{\mathrm{L}} \sin \varepsilon / \hbar$, where $\phi_{\mathrm{L}}$ is the colatitude angle of the place where the experiment was performed. This also indicates by how many parameters the wave function is influenced, which will become clear when more precise and more quantum complete measurements are performed.

Numerous neutron interference experiments have been performed to prove the analogy between light- and matterwave optics and to establish neutron quantum optics as a new tool for fundamental and applied research. In this connection, the coherence properties of the matter wavefield have to be elucidated in more detail. The coherence function, introduced in Eq. (1), is defined by the auto-correlation function of the overlapping wave functions, as

$$
\Gamma(\boldsymbol{\Delta})=\left\langle\psi^{*}(0) \cdot \psi(\boldsymbol{\Delta})\right\rangle .
$$

Fig. 5: Neutron wave packets in ordinary and momentum space at low and high interference orders, indicating the appearance of Schroedinger cat-like-states when the coherent shift of the wave packets exceeds the coherence length.
Fig. 4: Various Aharonov-Bohm effects for electrons and neutrons. Either a charge is transported coherently around a magnetic field or a magnetic moment is transported coherently around an electric charge (left), or the electric or magnetic interaction acts in a time-dependent fashion only (right)

When the spatial phase shift $\Delta_{\mathrm{i}}$ becomes much larger than the coherence lengths $\Delta_{\mathrm{i}}^{\mathrm{c}}$, the interference patterns disappear at high order, as stated above. But recent experiments have demonstrated that interference phenomena can be retrieved by proper post-selection procedures, even when the overall beam does not exhibit any interference pattern because there is no overlap of the wave packets at the third interferometer plate. This can be seen (Fig. 5) when the amplitude function of the wave packets are plotted

$$
\mathrm{I}(\mathrm{x}) \propto|\psi(\mathrm{x})+\psi(\mathrm{x}+\Delta)|^{2} .
$$

In this case, the interference pattern disappears, but in momentum space a significant spectral modulation appears:

$$
\begin{array}{r}
I(k) \propto \exp \left[-\left(k-k_{0}\right)^{2} / 2 \delta k^{2}\right] . \\
{\left[1+\cos \chi \frac{k_{0}}{k}\right]}
\end{array}
$$

as shown in Fig. 5 and experimentally verified by a proper momentum post-selection
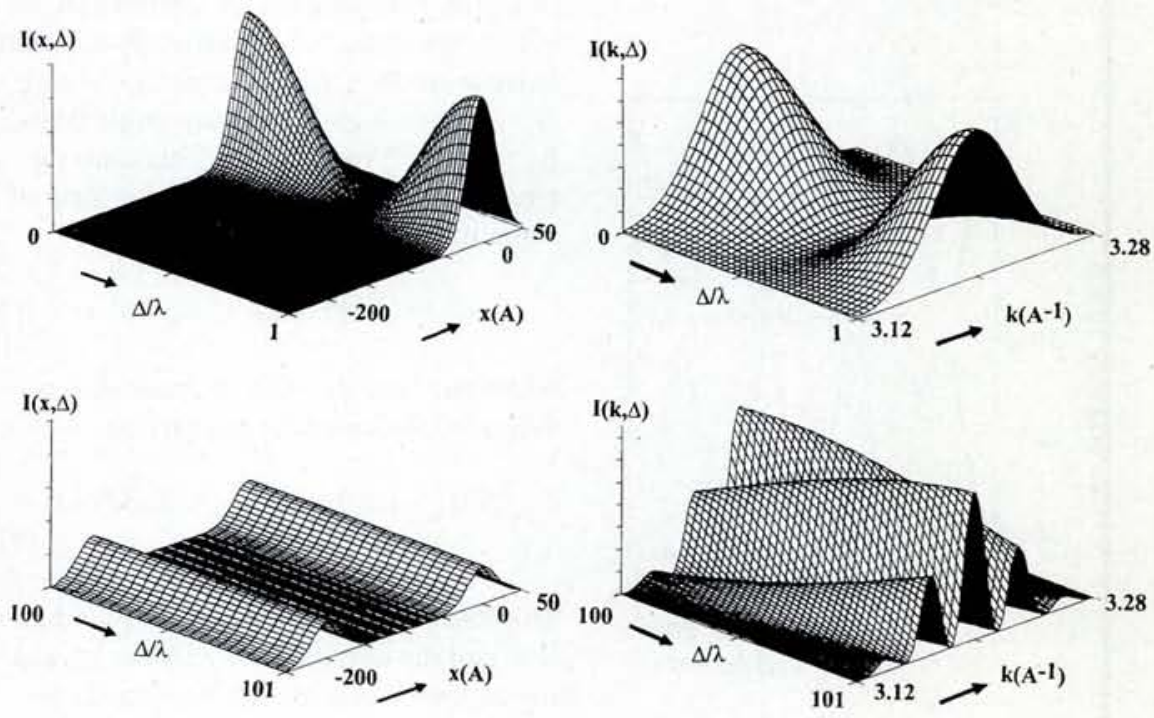

procedure using an additional analyzer crystal behind the interferometer [10] (Fig. 6). Superposition states of two macroscopically distinguishable states appear, namely, stationary Schrödinger cat-like states, exhibiting non-classical features such as squeezing, and entanglement, including the dressed neutron phenomenon as well [11]. These states are separated in ordinary space but oscillatory in momentum space and seem to be extremely fragile and sensitive to any kind of dephasing effects. Therefore, this kind of experiment is related to the recent progress in quantum optics and quantum measurement theory [12]. The experimental results show that interference phenomena can be exchanged between various parameter spaces and that they can be restored to the original parameter space. The results also show that a retrieval becomes more and more difficult after quantum state separation occurs and that a complete retrieval becomes impossible, in principle, due to various unavoidable quantum uncertainties and fluctuation effects.

Neutron interference experiments have shown that not only expectation values are measurable quantities, but that several properties of single particle wave functions are measurable as well. A detailed analysis also shows that the whole history of each particle is stored in its wave function and becomes measurable if experiments with sufficiently high resolution become feasible. In many aspects, neutron interferometry has contributed substantially to the understanding of the wave-particle duality of various topological phenomena and has pushed the sensitivities up to the quantum limits. The advent of atomic and molecular interfe- 
Fig. 6: Set-up for momentum post-selection experiments. By means of a momentum band filter (analyzer single crystal), interference fringes can be retrieved even when the contrast of the overall beam vanishes and the characteristic modulation of the momentum distribution can be observed, indicating non-classical neutron states.

rometry have also shown that there is no natural boundary between the micro and macro world and that it depends on human intuition and experimental effort up to which level quantum phenomena become visible. All experiments have shown that quantum theory is still a valid theory, and one can appreciate the pioneering work of the founders of this basic theory, who created it with so little experimental evidence. Now one has much more direct evidence - even on macroscopic scales - but, nevertheless, one notices that the interpretation of quantum mechanics in many cases goes beyond human intuition. Yet when more complete quantum experiments become feasible, the mysteries of quantum mechanics may be reduced step by step.

\section{References}

[1] H. Rauch, W. Treimer, U. Bonse; Phys. Lett. A47 (1972) 369

[2] U. Bonse, M. Hart; Appl. Phys. Lett. 6 (1965) 155 [3] C.S.Adams, M. Sigel, J. Mlynek; Phys. Rep. 240 (1994) 143

[4] H. Rauch, A. Zeilinger, G. Badurek, A. Wilfing, W. Bauspiess, U. Bonse; Phys. Lett. A54 (1975) 425 and S.A. Werner, R. Colella, A.W. Overhauser, C.F. Eagen; Phys. Rev. Lett. 35 (1975) 1053 [5] J. Summhammer, G. Badurek, H. Rauch, U. Kischko, A. Zeilinger; Phys. Rev. A27 (1983) 2523 and G. Badurek, H. Rauch, J. Summhammer; Phys. Rev. Lett. 51 (1983) 1015

[6] G. Badurek, H. Rauch, D. Tuppinger; Phys. Rev. A34 (1986) 2600

[7] Y. Aharonov, D. Bohm; Phys. Rev. 115 (1959) 485 and M.V.Berry; Proc. R. Soc. London A392 (1984) 45

[8] G. Badurek, H. Weinfurter, R. Gaehler, A. Kollmar, S. Wehinger, A. Zeilinger; Phys. Rev. Lett. 71 (1993) 307

9] R. Colella, A.W. Overhauser, S.A. Werner; Phys. Rev. Lett. 34 (1975) 1472 and J.L. Staudenmann, S.A. Werner, R. Colella, A.W. Overhauser; Phys. Rev. A21 (1980) 1419

[10]H. Rauch; Phys. Lett. A173 (1993) 240 and D.L. Jacobson, S.A. Werner, H. Rauch; Phys. Rev. A49 (1994) 3194

[11] E. Muskat, D. Dubbers, O. Schaerpf; Phys. Rev. Lett. 58 (1987) 2047

[12] W. Schleich, M. Pernigo, F.L. Kien; Phys. Rev. A44 (1991) 2172 and H. Zurek; Phys. Today, Oct. 1991, p. 36

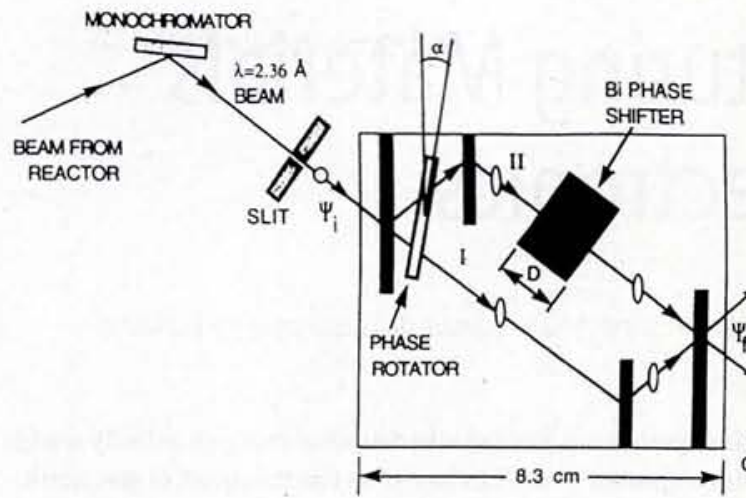

SKEW-SYMMETRIC NEUTRON NTERFEAOMETER

\section{UNFILTERED}

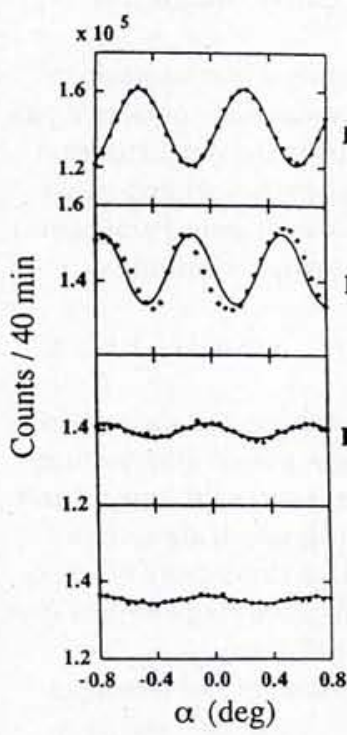

FILTERED

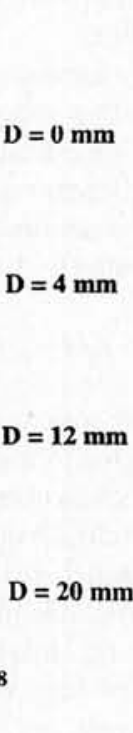

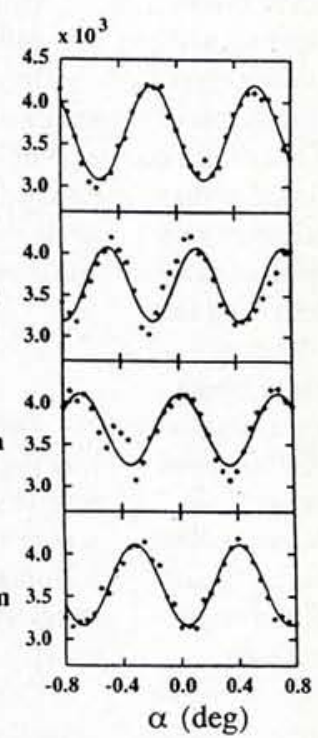
DISTRIBUTION
SPECTRAL

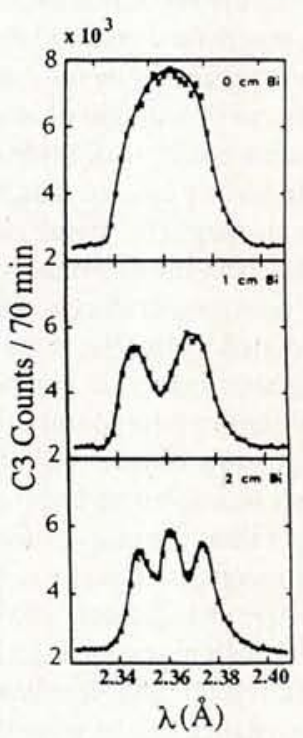

The forthcoming deadline for applications for magnet time allocation (September 1997 to January 1998) at the

\section{Grenoble high magnetic field laboratory}

is May 23, 1997.

Scientists of EU countries and Associated States (Iceland, Israel, Liechtenstein, Norway) are entitled to apply under the "Access to research under high magnetic fields" programme. Application forms are available on request.

Please contact: J.C. VALLIER

Laboratoire des Champs Magnétiques Intenses,

Max-Planck-Institut für Festkörperforschung ct

Centre National de la Recherche Scientifique

B.P. 166

38042 Grenoble Cedex 9 - France

Tel.: 04.76.88.10.01- Fax: 04.76.85.56.10

Bitnet: vallier@polycnrs-grc-fr 